\title{
UPPER SEMI-FREDHOLM AND KATO SPECTRUM OF AN $\alpha$-TIMES INTEGRATED SEMIGROUP
}

\author{
HAMID BOUA
}

\begin{abstract}
Let $(T(t))_{t \geq 0}$ be an $\alpha$-times integrated semigroup with generator $A$ on a Banach space $X$. In this paper, we show that the spectral mapping theorem holds for upper semi-Fredholm spectrum and we will give some consequences of this result. We also give an application on the Schrödinger operator.
\end{abstract}

\section{INTRODUCTION}

Let $X$ be a complex Banach space, $\mathcal{B}(X)$ denote the algebra of all bounded linear operators on $X$ and $\mathcal{C}(X)$ the set of all closed linear operators from $X$ to $X$. We denote by $D(A), R(A), N(A), \sigma(A), \sigma_{p}(A), \sigma_{r}(A)$ and $\sigma_{a p}(A)$, respectively, the domain, the range, the kernel, the spectrum, the point spectrum, the residual spectrum and the approximate point spectrum of an operator $A \in \mathcal{C}(X)$, (see [6]).

Next, $A \in \mathcal{C}(X)$ is called an upper semi-Fredholm if the range $R(A)$ is closed and $\operatorname{dim} N(A)<\infty$. The upper semi-Fredholm spectrum of $A$ is defined by (see e.g. [12])

$$
\sigma_{u f}(A)=\{\lambda \in \mathbb{C}: \lambda-A \text { is not upper semi-Fredholm }\} .
$$

Recall that a closed operator $A$ is said to be a Kato operator or semi-regular if $R(A)$ is closed and $N(A) \subseteq R^{\infty}(A)$. The Kato spectrum of $A$ is defined by

$$
\sigma_{k}(A)=\{\lambda \in \mathbb{C}: A-\lambda \text { is Kato }\} .
$$

We say that an operator $A \in \mathcal{C}(X)$ is essentially Kato or essentially semi-regular if $R(A)$ is closed and there exists a finite-dimensional subspace $F \subseteq X$ such that $N(A) \subseteq$ $R^{\infty}(A)+F$. The essentially Kato spectrum of $A$ is defined by

$$
\sigma_{e k}(A)=\{\lambda \in \mathbb{C}: A-\lambda \text { is essentially Kato }\} .
$$

For more information see [1] and [4].

An operator $A$ is said to have a single valued extension property at $\lambda_{0} \in \mathbb{C}$ (SVEP) if for every open disc $D_{\lambda_{0}} \subseteq \mathbb{C}$ centered at $\lambda_{0}$, the only analytic function $f: D_{\lambda_{0}} \longrightarrow D(A)$ which satisfies the equation $(A-z I) f(z)=0$ for all $z \in D_{\lambda_{0}}$ is the function $f \equiv 0$. $A$ is said to have the SVEP if $A$ has the SVEP for every $\lambda \in \mathbb{C}$ (see [2]). Denote by

$$
\mathcal{S}(A)=\{\lambda \in \mathbb{C}: A \text { has not the SVEP at } \lambda\} .
$$

Note that, $\mu \in S(A)$ if and only if there exists a sequence $\left(x_{i}\right)_{i \geq 0} \subseteq D(A)$ not all of them equal to zero such that $(A-\mu) x_{i+1}=x_{i}$ with $x_{0}=0$ and $\sup _{i \geq 1}\left\|x_{i}\right\|^{\frac{1}{i}}<\infty$, (see [7]).

Let $(S(t))_{t>0}$ be a strongly continuous, uniformly exponentially bounded family of bounded linear operators on a Banach space $X$. Let $A: D(A) \rightarrow X$ be a closed linear

2020 Mathematics Subject Classification. 47D03, 47A10, 47A11.

Key words and phrases. $\alpha$-times integrated semigroup, upper semi-Fredholm, Kato, essentially Kato, SVEP. 
operator with domain $D(A) \subseteq X$. If there are $\omega, \alpha \geq 0$ such that $\lambda$ is in the resolvent set of $A$ for all $\lambda$ with $\operatorname{Re}(\lambda)>\omega$ and the resolvent of $A$ is given by

$$
R(\lambda, A) x=\lambda^{\alpha} \int_{0}^{\infty} e^{-\lambda s} S(s) x d s \quad \text { for all } \quad x \in X,
$$

then $A$ generates $(S(t))_{t \geq 0}$ as an $\alpha$-times integrated semigroup on $X$ (see e.g. [5]).

W. Arendt showed that if $A \in \mathcal{C}(X)$ generates $T(t)$ as an $n$-times integrated semigroup, with $n \in \mathbb{N}$, then the Abstract Cauchy Problem $u^{\prime}(t)=A u(t), u(0)=x$ has a classical solution for all $x \in D\left(A^{n+1}\right)$. The notion of $\alpha$-times integrated semigroup for arbitrary $\alpha>0$, was introduced by M. Hieber in [11]. He showed in [10] and [11] that the functional analytic framework of $\alpha$-times integrated semigroups can be used to describe Abstract Cauchy Problems of certain differential operators on $L^{p}\left(\mathbb{R}^{n}\right)$.

In [5], C. R. Day studied the point, the approximate point and residual spectra of the $\alpha$-times integrated semigroups. In order to understand the behavior of the solutions in terms of the data concerning $A$, one seeks information about spectra of $T(t)$ in terms of spectra of $A$ (see $[5,9])$. In the same direction, we continue the development of the spectral theory of integrated semigroups.

In this paper, we show that if $(T(t))_{t \geq 0} \subset \mathcal{B}(X)$ is an $\alpha$-times integrated semigroup with generator $A$, then

$$
\sigma_{u f}(T(t)) \cup\{0\}=\left\{\int_{0}^{t} \frac{s^{\alpha-1}}{\Gamma(\alpha)} e^{\lambda s} d s, \lambda \in \sigma_{u f}(A)\right\} \cup\{0\} .
$$

We also prove that

$$
\left\{\int_{0}^{t} \frac{s^{\alpha-1}}{\Gamma(\alpha)} e^{\lambda s} d s, \lambda \in \sigma_{\star}(A)\right\} \cup\{0\} \subseteq \sigma_{\star}(T(t)) \cup\{0\},
$$

where $\star \in\{k, e k\}$. Moreover, we have equality if $A$ has the SVEP.

\section{MAin RESULtS}

We begin by the following lemmas.

Lemma 1. Let $(T(t))_{t>0}$ be an $\alpha$-times integrated semigroup with generator $A$ on Banach space $X$. Then, for all $\lambda \in \mathbb{C}, t \geq 0$ and $n \in \mathbb{N}$,

$$
\begin{aligned}
& \text { 1. }\left(\int_{0}^{t} \frac{s^{\alpha-1}}{\Gamma(\alpha)} e^{\lambda s} d s-T(t)\right)^{n} x=(\lambda-A)^{n} D_{\lambda}^{n}(t) x \text {, for all } x \in X . \\
& \text { 2. }\left(\int_{0}^{t} \frac{s^{\alpha-1}}{\Gamma(\alpha)} e^{\lambda s} d s-T(t)\right)^{n} x=D_{\lambda}^{n}(t)(\lambda-A)^{n} x \text {, for all } x \in D\left(A^{n}\right) . \\
& \text { 3. } R^{\infty}\left(\int_{0}^{t} \frac{s^{\alpha-1}}{\Gamma(\alpha)} e^{\lambda s} d s-T(t)\right) \subseteq R^{\infty}(\lambda-A) . \\
& \text { 4. } N(\lambda-A)^{n} \subseteq N\left(\int_{0}^{t} \frac{s^{\alpha-1}}{\Gamma(\alpha)} e^{\lambda s} d s-T(t)\right)^{n} .
\end{aligned}
$$

Proof. Clearly, $D_{\lambda}(t)$ is a bounded linear operator on $X$. Moreover (see [5]), we have

$$
\begin{aligned}
\left(\int_{0}^{t} \frac{s^{\alpha-1}}{\Gamma(\alpha)} e^{\lambda s} d s-T(t)\right) x & =(\lambda-A) D_{\lambda}(t) x, \text { for all } x \in X \\
& =D_{\lambda}(t)(\lambda-A) x, \text { for all } x \in D(A) .
\end{aligned}
$$

Proceeding by induction, we get the desired result. The assertions 3 and 4 follow easily from 1 and 2 .

Lemma 2. [5, Lemma 2.2] Let $(T(t))_{t \geq 0}$ be an $\alpha$-times integrated semigroup with generator $A$. For $\mu \neq 0, A$ is bounded on $\operatorname{ker}(T(t)-\mu)$ for any $t \geq 0$. 
Lemma 3. [5, Theorem 2.6] Let $(T(t))_{t \geq 0}$ be an $\alpha$-times integrated semigroup with generator $A$. Then, for all $t \geq 0$

$$
\sigma_{a p}(T(t)) \cup\{0\}=\left\{\int_{0}^{t} \frac{s^{\alpha-1}}{\Gamma(\alpha)} e^{\lambda s} d s, \lambda \in \sigma_{a p}(A)\right\} \cup\{0\} .
$$

We now state the general spectral mapping theorem for upper semi-Fredholm spectrum.

Theorem 1. Let $(T(t))_{t \geq 0}$ be an $\alpha$-times integrated semigroup with generator $A$. Then

$$
\sigma_{u f}(T(t)) \cup\{0\}=\left\{\int_{0}^{t} \frac{s^{\alpha-1}}{\Gamma(\alpha)} e^{\lambda s} d s, \lambda \in \sigma_{u f}(A)\right\} \cup\{0\} .
$$

Proof. Let $\mu=\int_{0}^{t} \frac{s^{\alpha-1}}{\Gamma(\alpha)} e^{\lambda s} d s \notin \sigma_{u f}(T(t))$, then $\operatorname{dim} N(\mu-T(t))<\infty$ and $R(\mu-T(t))$ is closed. From Lemma 1, we have $\operatorname{dim} N(A-\lambda)<\infty$. Now, we prove that $R(A-\lambda)$ is closed. Since $N(\mu-T(t))$ is finite dimensional, there exists a closed subspace $Y$ of $X$ such that $N(\mu-T(t)) \oplus Y=X$. But, $(\lambda-A)(N(\mu-T(t))) \cap D(A))$ is finite dimensional and therefore closed. Hence, we need only to show that $(\lambda-A)(Y \cap D(A))$ is closed.

Indeed, from the closed-graph theorem and the closedness of $R(\mu-T(t))$, it follows that there is a constant $C>0$ such that $\|(\mu-T(t)) x\| \geq C\|x\|$ for all $x \in Y$. From Lemma 1, we obtain that for every $x \in D(A),\|(\mu-T(t)) x\| \leq M\|(\lambda-A) x\|$ for some $M>0$.

The combination of the last two inequalities gives us

$$
\|(\lambda-A) x\| \geq \frac{C}{M}\|x\| \text { for all } x \in Y \cap D(A) .
$$

So that $R(\lambda-A)$ is closed. Consequently, $\lambda-A$ is upper semi Fredholm.

Conversely, let $\mu \in \sigma_{u f}(T(t)) \backslash\{0\}$ and $F=N(\mu-T(t))$. Then, $F$ is $A$-invariant and $T(t)$-invariant closed subspace of $X$. By Lemma $2, A_{\mid F}$ is a bounded operator generates the $\alpha$-times integrated semigroup $\left(T(t)_{\mid F}\right)_{t \geq 0}$, which yields $\sigma_{u f}\left(A_{\mid F}\right) \neq \emptyset$. Now, let $\lambda_{0} \in \sigma_{u f}\left(A_{\mid F}\right)$, according to the first implication of this theorem, we obtain

$$
\int_{0}^{t} \frac{s^{\alpha-1}}{\Gamma(\alpha)} e^{\lambda_{0} s} d s \in \sigma_{u f}\left(T(t)_{\mid F}\right)=\sigma\left(T(t)_{\mid F}\right)=\{\mu\} .
$$

Thus $\mu=\int_{0}^{t} \frac{s^{\alpha-1}}{\Gamma(\alpha)} e^{\lambda_{0} s} d s$. We prove that $\lambda_{0} \in \sigma_{u f}(A)$. Indeed, suppose that $A-\lambda_{0}$ is upper semi Fredholm, then $\operatorname{dim} N\left(\lambda_{0}-A\right)<\infty$ and $R\left(A-\lambda_{0}\right)$ is closed. Since $N\left(\lambda_{0}-A_{\mid F}\right)=D(A) \cap N\left(\lambda_{0}-A\right) \subseteq N\left(\lambda_{0}-A\right)$, then $\operatorname{dim} N\left(\lambda_{0}-A_{\mid F}\right)<\infty$.

Next, let $\left(x_{n}\right)_{n \geq 0}$ be a sequence of elements of $D(A) \cap F$ such that $\left(\lambda_{0}-A_{\mid F}\right) x_{n} \longrightarrow y$. Since $R\left(\lambda_{0}-A\right)$ is closed, there exists $x \in D(A)$ such that $y=\left(\lambda_{0}-A\right) x$. As $D_{\lambda_{0}}(t)$ is bounded, then $D_{\lambda_{0}}(t)\left(\lambda_{0}-A_{\mid F}\right) x_{n} \longrightarrow D_{\lambda_{0}}(t)\left(\lambda_{0}-A\right) x$, which give $(\mu-T(t)) x_{n} \longrightarrow$ $(\mu-T(t)) x$. Hence $(\mu-T(t)) x=0$. Consequently, $x \in F$ and $y \in R\left(\lambda_{0}-A_{\mid F}\right)$, which is absurd.

Theorem 2. Let $(T(t))_{t \geq 0}$ be an $\alpha$-times integrated semigroup with generator $A$. Then

$$
\left\{\int_{0}^{t} \frac{s^{\alpha-1}}{\Gamma(\alpha)} e^{\lambda s} d s, \lambda \in \sigma_{\star}(A)\right\} \cup\{0\} \subseteq \sigma_{\star}(T(t)) \cup\{0\} \quad \text { for all } \quad t \geq 0,
$$

with $\star \in\{k, e k\}$.

Proof. Let $\mu \in \sigma_{a p}(T(t)) \cup\{0\} \backslash \sigma_{\star}(T(t)) \cup\{0\}$. From Lemma 3, there exists $\lambda \in \sigma_{a p}(A)$ such that $\mu=\int_{0}^{t} \frac{s^{\alpha-1}}{\Gamma(\alpha)} e^{\lambda s} d s$. Hence, $M=R^{\infty}(\mu-T(t))$ is closed and $A$-invariant. 
Moreover, [5, Proposition 1.5], implies that $\tilde{T}(t)=T(t) / M$ is an $\alpha$-times integrated semigroup with generator $\tilde{A}=A / M$ on $X / M$. If $\mu-T(t)$ is semi-regular, it follows from Lemma 1 that

$$
N(\lambda-A) \subseteq N(\mu-T(t)) \subseteq R^{\infty}(\mu-T(t)) \subseteq R^{\infty}(\lambda-A) .
$$

Furthermore, from [8, Theorem 12.21], $\mu-\tilde{T}(t)$ is bounded below, which means that $\lambda-\tilde{A}$ is bounded below by Lemma 3. Now, if $\mu-T(t)$ is essentially semi-regular, then there exists a finite-dimensional subspace $F \subseteq X$ such that $N(\mu-T(t)) \subseteq R^{\infty}(\mu-$ $T(t))+F$. Hence

$$
N(\lambda-A) \subseteq N(\mu-T(t)) \subseteq R^{\infty}(\mu-T(t))+F \subseteq R^{\infty}(\lambda-A)+F .
$$

Furthermore, by [8, Theorem 21.7], $\mu-\tilde{T}(t)$ is upper semi Fredholm and by Theorem 1 $\lambda-\tilde{A}$ is upper semi Fredholm. In both cases $R(\lambda-\tilde{A})$ is closed. So it is sufficient to prove that $R(\lambda-A)$ is closed. Now, let $(\lambda-A) x_{n} \longrightarrow y$, there exists $\hat{a} \in X / M$ such that $(\lambda-\tilde{A}) \hat{x}_{n} \longrightarrow \hat{y}=(\lambda-\hat{A}) \hat{a}$. Therefore $y-(\lambda-A) a \in M \subseteq R(\lambda-A)$, this implies that $y \in R(\lambda-A)$. Consequently $\lambda \notin \sigma_{\star}(A)$, which ends the proof.

The following question arises in a natural way from our result.

Question 1. Do the inclusions of this Theorem are strict?

Proposition 1. Let $(T(t))_{t \geq 0}$ be an $\alpha$-times integrated semigroup with generator A. Then

$$
\mathcal{S}(T(t)) \subseteq\left\{\int_{0}^{t} \frac{s^{\alpha-1}}{\Gamma(\alpha)} e^{\lambda s} d s, \lambda \in \mathcal{S}(A)\right\} .
$$

In particular, if $A$ has the $S V E P$, then $T(t)$ has the $S V E P$ for all $t \geq 0$.

Proof. Let $\mu \in \mathcal{S}(T(t))$, by [5, Theorem 3.9], there exists $\lambda_{0} \in \sigma_{a p}(A)$ such that $\mu=$ $\int_{0}^{t} \frac{s^{\alpha-1}}{\Gamma(\alpha)} e^{\lambda_{0} s} d s$. This implies there exists a sequence $\left(x_{i}\right)_{i \geq 0} \subseteq X$ such that $x_{i}=(\mu-$ $T(t)) x_{i+1}$, where $x_{0}=0$ and $\sup _{i \geq 1}\left\|x_{i}\right\|^{\frac{1}{2}}<\infty$. We put $y_{0}=0$ and $y_{i}=D_{\lambda_{0}}^{i}(t) x_{i}$ for all $i \geq 0$, then $\left(y_{i}\right)_{i \geq 0} \subseteq D(A)$ and we have

$$
\begin{aligned}
\left(\lambda_{0}-A\right) y_{i} & =\left(\lambda_{0}-A\right) D_{\lambda_{0}}^{i}(t) x_{i} \\
& =D_{\lambda_{0}}^{i-1}(t) x_{i-1} \\
& =y_{i-1} .
\end{aligned}
$$

Then $\left\|y_{i}\right\|=\left\|D_{\lambda_{0}}^{i}(t) x_{i}\right\| \leq\left\|D_{\lambda_{0}}^{i}(t)\right\|\left\|x_{i}\right\| \leq M^{i}\left\|x_{i}\right\|$ and $\sup _{i \geq 1}\left\|y_{i}\right\|^{\frac{1}{i}} \leq M \sup _{i \geq 1}\left\|x_{i}\right\|^{\frac{1}{i}}<\infty$, which implies that $\lambda_{0} \in \mathcal{S}(A)$.

Remark 1. P. Aiena proved that if a bounded linear operator $A$ has the SVEP, then $\sigma_{k}(A)=\sigma_{a p}(A)$ and $\sigma_{e k}(A)=\sigma_{u f}(A)$. By using the same proof in [1, Corollary 2.45] and [1, Corollary 3.53], we can easily shows that our results remain true in the case of a closed linear operator. Therefore, the inclusion of the Theorem 2 will become equality when the generator $A$ has the SVEP.

Example 1. Take $A f=f^{\prime}$ on $X=C[0,1]$ with domain $D(A)=\left\{f \in C^{1}([0,1])\right.$ : $f(1)=0\}$. The once integrated semigroup generated by $A$ is given by the operators $S_{t} f(x)=\int_{x}^{m} f(s) d s$, where $m=\min \{1, x+t\}$. We can easily show that $\sigma(A)=\emptyset$. Then $\sigma_{\star}\left(S_{t}\right)=\{0\}$ with $\star \in\{u f, k, e k\}$.

Example 2. Consider the Schrödinger operator $A=i . \Delta$ on $L^{p}(\mathbb{R})$ for $p \geq 1$. A generates an integrated semigroup $(T(t))_{t \geq 0}$ given by $f \mapsto \mathcal{F}^{-1}\left(u_{t} \mathcal{F} f\right)$, with $u_{t}(\xi)=\int_{0}^{t} e^{-i s|\xi|^{2}} d s$. 
It is well known that $\sigma(A)=i \mathbb{R}^{-}$. Then $A$ has the SVEP and from Proposition 1, $T(t)$ has the SVEP for all $t>0$. According to the previous results, we obtain

$$
\sigma_{\star}(T(t)) \backslash\{0\}=\left\{\frac{e^{i r t}-1}{i r}: r \leq 0\right\}
$$

with $\star \in\{u f, k, e k\}$.

Acknowledgments. The author is thankful to the referee for his valuable comments and suggestions.

\section{REFERENCES}

1. P. Aiena, Fredholm and Local Spectral Theory with Applications to Multipliers, Kluwer Academic Publishers, 2004.

2. P. Aiena, C. Trapani, and S. Triolo, SVEP and local specral radius formula for unbounded operators, Filomat 28 (2014), no. 2, 263-273.

3. W. Arendt, C. J. K. Batty, M. Hieber, and F. Neubrander, Vector-valued Laplace Transforms and Cauchy Problems, Monographs in Mathematics, 96, Birkhäuser-Springer, Basel, 2011.

4. M. Benharrat et al., On the Kato, semi-regular and essentially semi-regular spectra, Functional Analysis, Approximation and Computation 6 (2014), no. 2, 9-22.

5. C. R. Day, Spectral mapping theorem for integrated semigroups, Semigroup Forum 47 (1993), no. 1, 359-372.

6. K. J. Engel and R. Nagel, One Parameter Semigroups for Linear Evolution Equations, SpringerVerlag, Berlin, 2000.

7. I. Erdelyi and Wang Shengwang, A Local Spectral Theory for Closed Operators, Cambridge University Press, London-New York-New Rochelle-Melbourne-Sydney, 1985.

8. Z. Garbouj and H. Skhiri, Essential ascent of closed operator and some decomposition theorems, Commun. Math. Anal. 16 (2014), no. 2, 19-47.

9. G. Greiner and M. Miiller, The spectral mapping theorem for integrated semigroups, Semigroup Forum 47 (1993), 115-122.

10. M. Hieber, Integrated semigroups and differential operators on $L^{p}$ spaces, Forum Math. 3 (1991), 595-612.

11. M. Hieber, Laplace transforms and $\alpha$-times integrated semigroups, Forum Math. 3 (1991), 595-612.

12. V. Williams, Closed Fredholm and Semi-Fredholm Operators, Essential Spectra and Perturbations, J. Functional Anal. 20 (1975), 1-25.

13. S. Č. Živković-Zlatanović, M. D. Cvetković, and D. S. Djordjević, On Closed Upper and Lower Semi-Browder Operators, Mediterr. J. Math. 12 (2014), no. 3, 1033-1045.

Mohammed First University, Pluridisciplinary Faculty of Nador, Nador, Morocco

E-mail address: hamid12boua@yahoo.com

Received 06/09/2019; Revised 12/10/2019 